\title{
Sobre el análisis de la «igualdad». El concepto de igualdad y el «momento feliz» de la transición española. Comentario a Alfonso Ruiz Miguel, Cuestiones de principios: entre política y Derecho
}

(2020) Centro de Estudios Políticos y Constitucionales Madrid, 615 pp.

\author{
Roberto Gargarella \\ Universidad de Buenos Aires \\ ORCID ID 0000-0003-1579-5427 \\ roberto.gargarella@gmail.com
}

\section{Cita recomendada:}

Gargarella, R. (2021). Sobre el análisis de la «igualdad». El concepto de igualdad y el «momento feliz» de la transición española. Comentario a Alfonso Ruiz Miguel, Cuestiones de principios: entre política y Derecho. Eunomía. Revista en Cultura de la Legalidad, 20, pp. 439-449.

doi: https://doi.org/10.20318/eunomia.2021.6088

Recibido / received: 01/02/2021

\section{Introducción}

En lo que sigue, examinaré algunas de las reflexiones en torno a la igualdad que aparecen en Cuestiones de principios, colección de textos publicada recientemente por el profesor Alfonso Ruiz Miguel (2020) ${ }^{1}$. El tema de la igualdad es abordado en el libro en diferentes pasajes pero, en particular ( $\mathrm{y}$ allí me centraré, conforme a la invitación que se me hiciera) el autor dedica a la cuestión, enteramente, la sección IV de su obra. Dicha sección IV se compone de 4 capítulos: el 16, dedicado a cuestiones conceptuales; el 17, que se refiere a temas -digamos así- político-partidarios (el liberalismo, la socialdemocracia, etc.); el 18, referido a la «igualdad política» (la democracia directa, la representativa, etc.); y el 19, relacionado con los «retos pendientes» en materia de «derechos de las mujeres». Como se ve, los temas de los que trata Ruiz Miguel en el área son muchos y muy variados; $y$, como puede anticiparse, el balance de lo que nos ofrece es muy rico. Concluimos nuestra lectura

\footnotetext{
1 El libro se compone de 23 ensayos, 21 de los cuales habían sido ya publicados con anterioridad. Cuando me refiera a algún pasaje de los mismos, citaré directamente la página a la que me refiero, conforme a la numeración que le corresponde dentro del libro.
} 
de la sección munidos de finas distinciones conceptuales; un «menú» amplio de problemas analíticos dispuestos sobre nuestra mesa de estudio; y aún, algunas discusiones y consideraciones «políticas» polémicas y provocativas. Con algunos de los capítulos mencionados encuentro un acuerdo casi completo (el 19, por ejemplo, que por dicha razón dejaré fundamentalmente de lado), a la vez que reconozco cierta incomodidad frente a algunas líneas fundamentales de su análisis. Como modo de homenajear al autor, y continuar nuestras conversaciones sobre una materia que nos apasiona a ambos, me concentraré en lo que sigue en unos pocos puntos que extraigo (algo caprichosamente) de la sección IV del libro, que son los que más me han motivado a replicar o tratar de responder sus argumentos. Me siento honrado por la oportunidad de comentar la obra de un amigo, a quien respeto y admiro por su trabajo, y a quien quiero agradecer también, por su bien sabida generosidad y amabilidad, de las que me he visto privilegiado. Pido disculpas de antemano por la (arbitraria) selección de temas que haré, y también, por poner el acento -mucho más- en los puntos de disenso que en los (muchos) puntos de acuerdo que mantengo con el autor.

\section{El concepto de igualdad: forma y sustancia}

Permítanme comenzar mi estudio del trabajo de Ruiz Miguel sobre la igualdad, a partir de una cita en apariencia extraña a su obra, que enseguida trataré de justificar. En su reseña del libro Sovereign Virtue. The Theory and Practice of Equality, de Ronald Dworkin, el profesor canadiense Will Kimlicka subraya la enorme potencia crítica del trabajo de Dworkin sobre la igualdad, a la vez que contrasta el vigor de las premisas del libro con las limitaciones propias de sus conclusiones. ${ }^{2}$ Al respecto, sostiene Kymlicka: «Encuentro decepcionante la conclusión de este volumen. La primera parte contiene una verdadera catarata de ideas teóricas novedosas e inspiradoras; la segunda parte aplica estas ideas excitantes de modos sorprendentemente convencionales» (2001, p. 136). Continúa Kymlicka: «Su principal interés en materia de políticas es el de mostrar que el estado de bienestar puede ser más "sensible a las elecciones", pero -sin embargo, agrega- Dworkin no invierte mucho tiempo o esfuerzo en pensar de qué modo el sistema puede ser más "insensible a las circunstancias". Y esto le da al volumen un (tal vez no buscado) sesgo conservador» (Ibíd.). Kymlicka concluye su reseña señalando que: "(Dworkin) no considera la posibilidad de que, en nuestro actual clima político, nuestros esfuerzos necesiten enfocarse de modos más innovadores en cómo terminar con las profundas y crecientes desigualdades en las circunstancias» (Ibíd.).

$\mathrm{Si}$, para introducir mi examen sobre lo sostenido por Ruiz Miguel en torno a la igualdad, tomo como punto de partida las reflexiones que hiciera Kymlicka sobre el

\footnotetext{
2 En Sovereign Virtue, Dworkin retoma y desarrolla los escritos que había ido desarrollando, durante años, en torno a la pregunta de What is Equality? En esencia, Dworkin distingue en su trabajo entre las «elecciones» de las personas -respecto de las cuales un Estado justo debe mostrarse particularmente «sensible»- y las "circunstancias» de las que las personas no son responsables (i.e., sus capacidades físicas, su color de piel, su género, etc.) y que el Estado justo no debe tomar como razón para beneficiar o penalizar a las personas ( $\mathrm{y}$ ellas no han elegido -no son responsables de- su raza, su color de piel, etc.!). Kymlicka asume a la siguiente como la proposición central del libro de Dworkin: «identificamos una distribución justa de los recursos imaginando una porción igual de recursos que es modificada a largo del tiempo, teniendo en cuento las elecciones de las personas en una subasta hipotética (que es sensible a las elecciones) y unas políticas de reaseguro hipotéticas (para protegerse contra circunstancias desiguales)» (2001, p. 135). Habitualmente, se entiende que la virtud de este esquema dworkiniano es que el mismo supera tanto a los enfoques tradicionales sobre la igualdad, que -de modo habitual- no se presentan como "sensibles a las elecciones" y descuidan el peso de las dotaciones naturales desiguales, entre las personas (i.e., sus talentos, etc.); como a las teorías libertarias y de derecha, que se centran exclusivamente en la cuestión de las elecciones personales, ignorando la necesidad de igualar a las personas en relación con las circunstancias que no controlan. Las conclusiones que agrega a sus trabajos iniciales no parecen, en efecto, derivadas necesariamente de las premisas de las que el libro parte.
} 
trabajo de Dworkin, ello es por las siguientes razones. En primer lugar, me apoyo en dicha reseña porque presentaré mis comentarios críticos sobre la igualdad desde un interés particular (como el que manifiesta Kymlicka) por la filosofía política, y una preocupación especial (también compartida con aquel) por las injustas desigualdades que caracterizan a nuestras sociedades. Y a la vez, estaré examinando las ideas sobre la igualdad de un autor -Ruiz Miguel- que -como nuestro célebre Dworkintambién se ocupa de hacer consideraciones sustantivas sobre la igualdad, a partir de una formación, y un interés profesional vinculado con la filosofía analítica y el análisis conceptual. En segundo lugar, considero -como considera Kymlicka sobre el trabajo de Dworkin- que un problema central en el estudio de Ruiz Miguel sobre la igualdad, es que el promisorio aparato analítico que presenta termina diluyéndose en una serie de consideraciones de alcance muy modesto, que en cierto modo desentonan con los puntos de partida. Y, en tercer lugar, parto del estudio de Kymlicka porque puedo decir sobre las conclusiones a las que llega Ruiz Miguel sobre la igualdad, algo similar a lo que sostuviera Kymlicka sobre las conclusiones del trabajo de Dworkin, y es que ellas le dan «al volumen un (tal vez no buscado) sesgo conservador».

Permítanme fundamentar las observaciones críticas introducidas aquí arriba, yendo directamente al estudio de la obra de Ruiz Miguel. Comenzaré por centrarme, de modo especial, en el capítulo número 16 del libro: "Concepto y concepciones de la igualdad» (capítulo que, no por azar, comienza con una referencia a Dworkin y a Kymlicka). Dicho capítulo es el más «denso» de los que aparecen en el libro, en materia de igualdad, junto con el texto sobre la «igualdad política» (que es el capítulo 18). En este capítulo 16, y como se adelanta desde su título, Ruiz Miguel se dedica a explorar el «concepto» y las "concepciones» de la igualdad (tomando como punto de partida la famosa distinción Dworkiniana entre ambas ideas). Allí, Ruiz Miguel utiliza varias páginas para precisar la distinción "concepto-concepciones», para lo cual recurre a la obra de los filósofos $\mathrm{H}$. Hart, J. Rawls y R. Dowrkin. Recién luego de tales aclaraciones (sobre "el concepto de concepto» y el "concepto de concepciones»), comienza a explorar específicamente el concepto de igualdad; se pregunta si hay uno o varios conceptos de igualdad; y refiere a los dos rasgos básicos que atribuye al concepto bajo estudio: su carácter «relacional», y su «dependencia de la noción de relevancia». Al examinar el carácter «relacional» de la igualdad, se interna en consideraciones sobre las ideas de «identidad», "semejanza» y «diferencia»; y al examinar la noción de «relevancia», considera también las funciones «descriptiva» y «prescriptiva» de igualdad; la igualdad «neutra» e «injusta» y (lo que denomina) la «supuesta asimetría» entre los conceptos de «igualdad» y «desigualdad». Finalmente, en la última parte del capítulo, Ruiz Miguel examina la naturaleza de la relación entre el concepto de igualdad y las concepciones que lo especifican, en donde vuelve a introducir algunas distinciones conceptuales (i.e., «juicios analíticos» y «sintéticos»).

Mi primera reacción frente al capítulo, es sugerir que el profesor Ruiz Miguel tiende a quedar atrapado en un pantano de distinciones conceptuales, en muchos casos -por lo demás- no esenciales para el tratamiento específico de la cuestión de igualdad, o los problemas derivados de ella y que interesan al autor del libro. Uno se pregunta, al leer sus clasificaciones en la materia: son tales distinciones esenciales para el estudio que se propone Ruiz Miguel en el libro (recuérdese que él se ocupa, en el mismo, de la socialdemocracia, del neoliberalismo, de la igualdad de género, de la igualdad política, etc.). ¿Cuánto aportan, tales distinciones iniciales, a la tarea (comprensiva, normativa, evaluativa) que sigue? ¿Entramos ahora en dichos análisis pertrechados de conceptos que nos permiten pensar con más claridad sobre tales asuntos? Por ejemplo, no parece tener mucho sentido dedicar un tercio del capítulo sobre el concepto de igualdad, a precisar la diferencia entre «concepto» y «concepciones»; o, luego, volver a la distinción entre «juicios analíticos» y 
«sintéticos»; o entrar en disquisiciones sobre la noción de «relevancia». Menos sentido tiene dicho análisis conceptual cuando las distinciones fatigosamente exploradas en el capítulo -según comprobamos al proseguir la lectura del libro- no terminan resultando imprescindibles para entender, por ejemplo, los usos más «políticos» que Ruiz Miguel explora, en torno a la idea de igualdad, en el capítulo 17; ni para entender la «igualdad política» que se examina en el capítulo 18: capítulo 18 en donde, por lo demás (y como veremos) el autor introduce toda una nueva oleada de distinciones conceptuales (igualdad "vertical», "horizontal», "de impacto», "de influencia») que no estaban presentes en el (conceptualmente denso) capítulo 16. Y finalmente, agregaría, el análisis conceptual del caso termina resultando todavía menos productivo, cuando el mismo, más que aportarnos una base imprescindible para entender el mundo que nos rodea, viene a complejizar al extremo o tornar más difícil (si no imposible, como veremos) dicho análisis.

Por supuesto -se podrá decir- es injusto enfocar críticamente el modo en que el profesor Ruiz Miguel encara el estudio de la igualdad, cuando sabemos que se trata de un destacadísimo profesor de filosofía del derecho; que tiene una amplia formación en la filosofía analítica; y que recordamos que él ha dedicado buena parte de su vida académica a trazar cuidadosas distinciones conceptuales. Sin embargo, recuérdese el referido caso de Ronald Dworkin -por tomar el ejemplo de un autor que parece inspirar buena parte de las reflexiones de Ruiz Miguel en el libro (al punto que su mismo título se emparienta directamente con el título de un libro de Dworkin, $A$ Matter of Principle)-: Dworkin también proviene de la filosofía analítica; también se interesa de modo especial por la filosofía del derecho; y también "ancla» sus análisis normativos en el examen detallado del concepto de igualdad (i.e., antes de partir a las aguas profundas de la igualdad de recursos). Y, sin embargo, uno reconoce en los escritos de Dworkin sobre la igualdad un trabajo analítico finísimo que es puesto inmediata y directamente al servicio de su filosofía política, y de su crítica a las injustas desigualdades que lo rodeaban (y no es fácil mostrar a tales conexiones como impropia o indebidamente fundadas). En efecto, el trabajo de Dworkin sobre la igualdad pretende servir como base para someter a crítica el estado presente del rule of law en los Estados Unidos, a la vez que ofrece un ideal regulativo igualitario desde el cual re-pensar y reconstruir ese «Estado de derecho».

En mi opinión, no es ese tipo de aproximación -dworkiniana- la que encontramos en el trabajo conceptual ofrecido por Ruiz Miguel, en torno a la noción de igualdad. El ejercicio resulta, en este caso -es mi opinión- menos atractivo: se trata de un emprendimiento teórico-formal, que complejiza al extremo nuestro conocimiento sobre conceptos y concepciones de la igualdad, pero que no nos ayuda, finalmente, a enriquecer nuestro entendimiento y nuestra capacidad crítica, en relación con las profundas injusticias y desigualdades que nos rodean (que no son pocos, agregaría, aún en lo que fuera el «momento feliz» de las transiciones democráticas europeas). Por el contrario $-y$ conforme veremos en el siguiente apartado- dicho análisis nos deja enfrentados a una complejísima madeja conceptual que amenaza con obstaculizar, quitar sentido o bloquear directamente nuestro análisis.

3. Del análisis formal al sustantivo; de la filosofía de los conceptos a la filosofía política

En el capítulo 18 de su libro, dedicado a la «igualdad política», Ruiz Miguel renueva -antes que "prosigue» o "profundiza»- su inicial estudio sobre el concepto de igualdad. El autor re-inicia, de algún modo, su análisis anterior (el del capítulo 16), que apoya aquí en una diversidad de distinciones conceptuales sobre la igualdad («igualdad vertical», «igualdad horizontal», «igualdad de impacto», «igualdad de 
influencia», «igualdad en los resultados», «igualdad en los procedimientos», «igualdad cuantitativa», «igualdad cualitativa», etc.). Tales distinciones, tributarias de la filosofía dworkiniana, terminan sin embargo por perder de vista -y así, por quitar centralidad- a la distinción nuclear que caracterizó al liberalismo igualitario de Dworkin y Rawls. En el capítulo 18, y por ejemplo, la preocupación por las "circunstancias desiguales» podría haber jugado un papel clave en la determinación de las condiciones de la ciudadanía política; o en la reflexión sobre los prerrequisitos de la democracia. Tales consideraciones podrían haberlo llevado a repensar más críticamente su acercamiento a las ideas de "democracia representativa» y «democracia directa»: ideas a las que dedica bastante espacio en el capítulo, pero sin aludir a los profundos y graves problemas que hoy ya constituyen el sentido común de las ciencias político-jurídicas (la «erosión democrática»; el «backsliding» democrático; la «muerte lenta» de la democracia; «la fatiga democrática»; etc., en por ejemplo, Ginsburg \& Huq, 2018; Graber et al., 2018; Levitsky \& Ziblatt, 2018). Mejor aún, Ruiz Miguel podría haber retomado las decenas de estudios realizados por Dworkin en torno a las implicaciones político-democráticas de la idea de igualdad: igualdad, democracia y control judicial; democracia e impuestos progresivos; democracia e igual trato en tiempos de terrorismo; etc. Se trata de temas que Dworkin analizó en repetidas ocasiones, y a los que dedicó buena parte de su vida académica, y en particular dos libros, que no aparecen abordados en el texto de Ruiz Miguel (uno ya citado, sobre la igualdad, Sovereign Virtue, publicado antes de la aparición del texto de Ruiz Miguel, y otro que Dworkin diera a luz con posterioridad a dicho texto, y referido más directamente a los problemas de la democracia: Is Democracy Possible Here?, Dworkin, 1996; Dworkin, 2000; Dworkin, 2006). Sin embargo, el estudio que realiza Ruiz Miguel no nos conduce a tales indagaciones, a la vez que reserva severas críticas al igualitarismo de Dworkin (al que acusa de «adornar con grandilocuentes ropajes idealistas la...pedestre igualdad política...de la que algunos podemos disponer», p. 489). Perdida en los laberintos de la «igualdad vertical», «de impacto», etc., el liberalismo igualitario que presenta Ruiz Miguel termina diciendo mucho menos de lo (mucho, interesante) que está llamado a decir. Más grave que eso -y aquí vuelvo a retomar la reseña de Kymlicka sobre Dworkin- el trabajo que presenta Ruiz Miguel sobre la igualdad resulta, en un sentido tal vez inesperado, conservador. Conservador, agregaría, de un modo más severo que el que aparecía siéndolo (según Kymlicka) la teoría de Dworkin. Y es que, si el libro de Dworkin terminaba por ocuparse mucho más de la igualdad «sensible a las elecciones» que de la igualdad «insensible a las circunstancias», el libro de Ruiz Miguel, más que reforzar una sola de ambas opciones (como parecía hacer Dworkin, según Kymlicka), abandona por completo dicha nuclear distinción (elecciones-circunstancias), que es reemplazada por otras, menos potentes -y menos necesarias, agregaría- en el capítulo. Ruiz Miguel termina por describir a la igualdad como un ideal que «no resulta...tan estricto e importante como podría parecer a simple vista» (2020, p. 489).

El tipo de problemas que señalo para el capítulo 18 estaban ya presentes en el mismo capítulo conceptual del que partíamos (el capítulo 16). Dicho capítulo concluía, de hecho, empujándonos a pensar la idea de igualdad como una «forma», desprovista de "sustancia», «vacía» y susceptible de ser «rellenada» caprichosamente, casi de cualquier modo. Cabe mencionar al respecto, y por caso, las breves consideraciones que el autor ofrecía, en dicho capítulo, en torno a la notable idea rawlsiana de las «arbitrariedades morales». Para los menos familiarizados con el trabajo de Rawls, recuerdo simplemente que la noción de «hechos moralmente arbitrarios» es la que le sirvió para señalar que una sociedad justa no podía tratar mejor o peor a las personas por hechos de los que las personas no eran responsables (su raza, su color de piel, etc.): una noción que siempre mostró 
tener una potencia crítica extraordinaria ${ }^{3}$. Sin embargo, la conclusión que propone Ruiz Miguel frente a dicha concepción tan influyente, que inauguró un nuevo paradigma en los estudios sobre la igualdad, resulta desalentadora. Nos dice el autor: "la cuestión está en determinar si las ideas de "arbitrariedad" o de "adecuación" son nociones (o formas) vacías rellenables de cualquier contenido, o si, aun siendo nociones abiertas, de todos modos no son por completo vacías porque establecen o presuponen límites dentro de los cuales es posible afirmar que existen ciertas relaciones relevantes de igualdad (o de desigualdad) y que, por más que puedan estar en conflicto según diferentes concepciones, a la vez, señalan algún perímetro fuera del cual no es posible afirmarlo» (2020, p. 428). Es decir, el núcleo de la teoría que cambió la historia del pensamiento igualitario termina presentado como una propuesta formal antes que sustantiva; radicalmente imprecisa, sino directamente vacía; y abierta a «cualquier contenido». Es allí -al advertir ese contraste-cuando pienso que el enfoque que propone Ruiz Miguel sobre la igualdad corre el riesgo de quedar encerrado en el propio laberinto conceptual en el que busca apoyarse: se trata de una propuesta que insiste sobre el análisis de conceptos, aún a riesgo de que perdamos de vista lo sustantivo de una teoría como la que nos ofrece Rawls ${ }^{4}$.

En definitiva, el análisis de Ruiz Miguel nos empuja a pensar que no hay nada demasiado preciso (¿ni relevante?) en la reflexión filosófica sobre la igualdad: se trataría de una idea formal, muy limitada -sino impotente- y más bien carente de significado. De hecho, es esa cruda conclusión la que parece desprenderse del último párrafo del capítulo 16, en donde Ruiz Miguel retoma y resume su análisis conceptual, con la ayuda de una cita de Fitzjames Stephen. Junto con Stephen, Ruiz Miguel nos dice que la «igualdad es una palabra tan amplia y vaga que por sí sola carece casi de significado» (donde el subrayado del "casi» es suyo, pero que no sirve para obviar la demoledora conclusión anterior [2020, p. 431]).

\section{Socialdemocracia, Europa y la teoría de Rawls}

Al estudiar el capítulo 17 de Cuestiones de Principios, que lleva por título «lgualdad, Liberalismo y Socialdemocracia», se reconocen algunas de las limitaciones "políticas», si se quiere- que se derivan del análisis conceptual sobre la igualdad presentado por Ruiz Miguel. En este nuevo texto, el profesor contrasta dos propuestas políticas, en apariencia en profunda tensión entre sí, siendo éstas el neoliberalismo y la socialdemocracia. Y considera que tales propuestas políticas (tan habitualmente enfrentadas como polos opuestos dentro del continuo de nuestro arco político) resultan, en verdad, más parecidas que diferentes (tan «lo mismo» resultan, que Ruiz Miguel debe aclarar dos veces, sobre el final del texto, que su examen no pretende

\footnotetext{
3. Por lo demás, cabe agregar que dicha propuesta de Rawls -justamente en razón de su potencia- ha resultado sobre-estudiada, y en tal sentido ha sido objeto de análisis y precisiones muy específicas. Se ha discutido muchísimo sobre los factores «moralmente arbitrarios» más determinantes en nuestras sociedades (raza, etnia, género, clase, etc.). Más todavía, podrá agregarse: Rawls revolucionó al pensamiento igualitario con un enfoque tal que nos invitó a pensar, en cierto sentido, yendo mucho más allá de lo que había sido en Karl Marx el punto de llegada. Ello así, a través de afirmaciones como la de que «nadie es dueño de sus talentos»; o que los talentos pertenecen a un «acervo común»; o que nadie puede pedir recompensas especiales por haber nacido dotado de una mayor rapidez mental o de más velocidad en las piernas.

${ }^{4} \mathrm{Al}$ respecto, me remitiría a lo dicho por Martha Nussbaum, en un breve escrito que dedicara a reflexionar sobre la obra de Rawls. Si una cosa destaca Nussbaum de todo el aporte rawlsiano, es el modo en que el mismo contribuyó a que la filosofía abandonara el confinamiento al que la había sometido el «positivismo lógico»: hasta entonces -nos dice Nussbaum- la filosofía aparecía encerrada en «la investigación empírica y el análisis conceptual» (Nussbaum, 2002). Rawls -concluye Nussbaum- ayudó a la filosofía política a encontrar su lugar, retomando las grandes preguntas sobre la justicia y la igualdad. Es este análisis -agregaría, teniendo en vista el trabajo de Ruiz Miguel- el que la filosofía no debería (volver a) abandonar, haciendo colapsar la filosofía política en el análisis conceptual.
} 
«identificar el neoliberalismo con la socialdemocracia» ni «difuminar hasta hacer desaparecer las diferencias» entre ambas posturas [2020, pp. 452-53]).

Aquí se advierten -como anticipaba- algunos problemas relacionados con el capítulo anterior (el 16), concentrado en el análisis conceptual. Las dificultades en las que pienso se derivan, en parte, del haber diluido conceptualmente el peso o valor de los componentes esenciales del liberalismo igualitario (por caso, la noción de «arbitrariedades morales»); y en parte, también, del haber optado por precisiones conceptuales sobre la igualdad que no parecen vincularse directamente con los usos que damos a tal concepto en nuestro lenguaje político cotidiano. Por ello, nos encontramos en este texto una comparación entre aparentes opuestos neoliberalismo, socialdemocracia- en donde, finalmente, los conceptos en tensión aparecen básicamente superpuestos -unos colapsados sobre los otros- y nada parece demasiado diferente de lo que tiene enfrente. La izquierda termina siendo algo muy parecido a la derecha; la socialdemocracia resulta un pariente cercano del neoliberalismo; el liberalismo igualitario se muestra como difícilmente distinguible del liberalismo conservador; y la dupla teórica Friedrich Hayek-Milton Friedman aparece hermanada con la de John Rawls y Ronald Dworkin.

Para ser justos con el autor, debe decirse, por un lado, que concepciones y teorías como las referidas (izquierda, derecha, liberalismo igualitario, liberalismo conservador) son en efecto complejas y guardan ambigüedades muchas veces buscadas. Por otro lado, debe dejarse en claro que Ruiz Miguel acerca estrechamente, pero no identifica, a esos polos en apariencia opuestos; y debe decirse también que el profesor español se ocupa de aclararnos que él interpreta las tesis centrales de autores como los citados (Rawls, Dworkin, Hayek, Friedman) «hasta llevarlas a conclusiones a las que ellos no se han adherido expresamente pero que no creo que fuercen su pensamiento» (2020, p. 450). Pero allí reside justamente el problema: en los hechos, Ruiz Miguel fuerza algunas de las tesis centrales de tales autores hasta extremos que ellos seguramente rechazarían, y que no parecería apropiado atribuirles. Es que -permítanme ponerlo así- sobrevuela al capítulo un aire de «fin de la historia» (propio, seguramente, de ese «momento feliz» en el que fue escrito), que lleva al autor a sugerir que la Europa occidental, con su «desplazamiento al centroderecha», representa la arena común de la política contemporánea.

Me preocupa en todo caso, y en particular, el modo en que, en el contexto de Cuestiones de Principios, la teoría de la justicia rawlsiana aparece «sin alma», descolorida y desprovista de su potencia igualitaria. De hecho, Ruiz Miguel parece suscribir una lectura difundida pero injusta, de la teoría de Rawls, que asimila a la misma con el moderado liberalismo económico que tiende a predominar en los Estados Unidos, condimentado con algunas pizcas de impuestos a las ganancias, más al estilo europeo. De allí que el profesor pueda acercar al pensamiento de Rawls al de Hayek, atribuyéndole al primero la justificación de «una cierta ampliación temporal de las desigualdades...para las situaciones de crecimiento económico»; «el aumento a largo plazo de las desigualdades siempre que, además, los grupos peor situados en cada momento fueran mejorando en períodos sucesivos» (2020, p. 451). Ruiz Miguel sugiere «una posible coincidencia parcial entre la posición de Rawls y la de los neoliberales» (en la medida en que fuera cierta una tesis que él parece acompañar como si fuera cierta), conforme a la cual «la mayor eficiencia económica termina produciendo mayor igualdad» (Ibíd.).

Disiento con la reconstrucción que propone Ruiz Miguel, y considero incorrecto sugerir que Rawls justificaría desigualdades tales. Mi disidencia se debe, seguramente, a que participo de la tendencia que lee a Rawls del modo exactamente inverso al propuesto por Ruiz Miguel: estoy entre aquellos que destacan los aspectos 
más igualitarios de la Teoría de la Justicia. A mi favor, en todo caso, he de decir que la lectura que propondría de Rawls parece fiel al propio texto de su obra magna; de sus consideraciones explícitas sobre los programas económicos que resultarían consistentes con su teoría (en donde Rawls se limita a hablar de dos alternativas, ambas muy radicales: la «democracia de propietarios» y el «socialismo de mercado»); y de las mismas aclaraciones que Rawls se viera obligado a hacer al respecto. Dos de tales aclaraciones resultan especialmente pertinentes, para este apartado.

La primera de tales aclaraciones ayuda a reconocer por qué no es correcto el apresuramiento que muestra Ruiz Miguel en convertir a la «teoría de la justicia» en la justificación teórica de una (complaciente) política socialdemócrata. Adviértase, por caso, las precisiones que realizara el mismo Rawls en su libro Justice as Fairness. $A$ Restatement (2001): un libro que el norteamericano dedicara exclusivamente a lidiar con las numerosas y furibundas críticas recibidas por su «teoría» original. En la página 139 de Justice as Fairness, Rawls hace un deliberado esfuerzo por distinguir -por impedir que se confunda- a su propuesta en favor de una «democracia de propietarios» con el "capitalismo de estado de bienestar»-welfare state capitalism (Rawls no se ocupa aquí, en cambio, de distinguir al segundo con su modelo económico alternativo, el "socialismo de mercado», porque en este caso las diferencias resultaron siempre más evidentes, ante la ausencia de propiedad privada de los medios de producción). Dice Rawls entonces, para contrastar el estado de bienestar y la «democracia de propietarios»:

Una diferencia mayor es la siguiente: las instituciones que están en el trasfondo de una democracia de propietarios (background institutions) trabajan para dispersar la propiedad de la riqueza y del capital, y así impedir que una porción pequeña de la sociedad termine por controlar la economía e, indirectamente, también la vida política. Por el contrario, en el capitalismo de estado de bienestar se permite que una pequeña clase tenga casi el monopolio de los medios de producción (2001, p. 139). ${ }^{5}$

Y aclara también Rawls (agregaría, de modo significativo frente a reconstrucciones como la propuesta en Cuestiones de Principios): «Los menos aventajados» no deben verse como «objetos de nuestra caridad y compasión, mucho menos de nuestra lástima, sino como aquellos a quienes les debemos reciprocidad como cuestión de justicia política» (2001, p. 139; ver también, en un sentido semejante, O'Neill \& Williamson, 2012). Precisiones como las anteriores, según entiendo, nos permiten entender bien las enormes distancias que existen (y que deben mantenerse) entre la visión sobre la economía y la desigualdad que subyace a la «teoría de la justicia», y la propuesta que pudiera avanzar la socialdemocracia europea -un régimen económico que Rawls no estaba dispuesto a suscribir, y mucho menos a justificar, por razones de fondo, «como una cuestión de justicia política».

Por otro lado -en relación con la segunda «aclaración rawlsiana» que quería traer a cuento- citaría un famoso intercambio epistolar entre Philippe Van Parijs y John Rawls, que el primero considerara, con razón, como «el texto más abiertamente anti-capitalista escrito por Rawls», y también (de particular relevancia para nuestros propósitos) como «el único escrito en el que Rawls trata, de modo explícito, acerca de la Unión Europea» (Van Parijs \& Rawls, 2003). Cito el texto en su idioma original,

\footnotetext{
${ }^{5}$ Continúa Rawls: «la democracia de propietarios evita esto, no a través de la redistribución del ingreso hacia aquellos con menos recursos al final de cada período -para decirlo de algún modo- sino asegurando una dispersión en la propiedad de los bienes productivos y el capital humano (esto es, la educación y las habilidades que surgen del entrenamiento) al comienzo de cada período, y todo esto contra un trasfondo de equitativa igualdad de oportunidades. Lo que se busca no es, simplemente, asistir a aquellos que pierden por razones de accidente o mala suerte (aunque esto debe hacerse), sino poner a todos los ciudadanos en una posición de controlar sus propios asuntos, desde un lugar de igualdad económica y social» (2001, p. 139).
} 
para que no se pierdan los términos extremadamente críticos que Rawls reservara para una Unión Europea entonces en vías de consolidación. Sostuvo Rawls, entonces:

The large open market including all of Europe is aim of the large banks and the capitalist business class whose main goal is simply larger profit. The idea of economic growth, onwards and upwards, with no specific end in sight, fits this class perfectly. If they speak about distribution, it is [al]most always in terms of trickle down. The longterm result of this — which we already have in the United States - is a civil society awash in a meaningless consumerism of some kind. I can't believe that that is what you want (Van Parijs \& Rawls, 2003).

Queda claro, de este modo, el profundo contraste existente entre el pesimismo de Rawls sobre los desarrollos políticos y sobre todo económicos que se afirmaban en Europa, y el optimismo de Ruiz Miguel al respecto. En todo caso, me importa que la discusión subyacente no quede como aludiendo a un mero contraste entre «pesimistas» y "optimistas» (tampoco se trata de una carta escrita alguna vez por John Rawls, sino de una en donde por fin dejó en claro la visión sobre la Unión Europea que no había expuesto en sus demás textos, pero que resulta por completo consistente con su teoría general). Las diferencias son de fondo, y refieren, finalmente, a concepciones sobre la justicia muy diversas.

5. Una cuestión de perspectivas: El «momento feliz» de la socialdemocracia española

El profesor Ruiz Miguel culmina su ensayo más sustantivo sobre la igualdad política -el 18- afirmando que el ideal en cuestión no parece ser «tan estricto e importante como podría parecer a simple vista», y admitiendo que su conclusión puede aparecer como «demasiado conformista con los sistemas democráticos existentes» (2020, p. 489). De modo similar, él cierra su ensayo sobre «lgualdad, liberalismo y socialdemocracia»-el capítulo 17- señalando que la posición que defiende en la materia «no desentona con las tendencias sociales dominantes hoy en Europa, como lo muestran la decisividad electoral del centro político y la alta expectativa...que una gran mayoría mantiene sobre la principal responsabilidad del Estado en materia social» (2020, p. 453).

Me pregunto por qué el profesor Ruiz Miguel tiende a concluir sus estudios sobre la igualdad con afirmaciones tan diferentes de aquellas por las cuales muchos comenzaríamos. Quiero decir, muchos empezaríamos el examen sobre la cuestión afirmando que el ideal de la igualdad es, ha sido, y seguirá siendo fundamental para la reflexión filosófico-política; y que nuestra situación en términos de igualdad política es alarmante, por muchas razones a todas luces evidentes y comprensibles, entre las que destacan la presencia de sistemas políticos dominados por elites, en donde la regla es la exclusión mayoritaria y la captura de los beneficios por unos pocos. Partiríamos de tales premisas, que seguramente nos llevarían a conclusiones muy diversas de aquellas a las que llega nuestro autor. Propongo dos razones que podrían ayudar a entender estos diferentes enfoques: la primera tiene que ver con el espacio y la segunda con el tiempo.

Sobre el espacio. Entiendo que, en cierta medida, las diferencias de enfoque que advierto entre el análisis que propone el profesor Ruiz Miguel sobre la igualdad, y el que muchos - desde contextos diferentes- propondríamos, pueden tener que ver con esta cuestión de perspectivas: valga decir, desde dónde miramos el problema. El contexto socio-político en el que cada uno de nosotros vive puede explicar, seguramente, parte de nuestros diferentes acercamientos a la cuestión: su relativa 
conformidad o acuerdo con lo que existe, frente al inconformismo que -desde aquí, por ejemplo, en América Latina- muchos mostramos, ante el estado de la vida sociopolítica. Pensar la «vida política» desde contextos muy marcados por las desigualdades injustas (nos) fuerza a muchos, seguramente, a «buscar ayuda» y respaldo en la idea de igualdad, con el objeto de cuestionar y someter a crítica el mundo político que nos rodea.

Sobre el tiempo. Pienso que Ruiz Miguel es uno de los grandes autores que nos ha dado la filosofía española de la transición democrática. Al decir «transición democrática» (española o europea) me refiero, fundamentalmente, a los años de bonanza y felicidad que trajo aparejado el gobierno del Partido Socialista encabezado por Felipe González, y que encontraron reflejo, también, en la «oleada socialdemocrática» que primó en Europa en los años 80. Un tiempo de optimismo, de libertades recuperadas, de más profunda, extendida y efectiva igualdad. Leo los análisis de filosofía política de Ruiz Miguel, y me es muy difícil sustraerme de la imagen de aquellos idílicos años, que prometían bajar a tierra «el maná» de la utopía igualitaria: una sociedad que aspiraba a asegurar a cada quien lo que sus necesidades le reclamaban. Con el correr del tiempo, aquellos años de ilusión sin horizontes, sin fin, se convirtieron en un recuerdo, y el Estado de Bienestar Europeo (y el español en particular) mostró sus límites y fisuras, que incluyeron crisis de gravedad (que hoy -en tiempos de pandemia- recrudecen), pero que no alcanzaron a eliminar del todo, la idea según la cual nos encontramos en un desafortunado paréntesis, de una realidad que, en verdad, tiende a parecerse a aquello que la socialdemocracia nos proponía. Desde tal perspectiva, por supuesto, las angustias por la igualdad que no alcanzamos, y las críticas a las desigualdades presentes, parecen exageradas. Alguien podría decir: «vamos, no estamos tan mal en realidad, ni falta tanto para cerrar este paréntesis conservador».

Explico, en definitiva, muchas de las diferencias que encuentro entre el análisis de la igualdad que sugiere Ruiz Miguel en su libro, y el que muchos -en cambiopropondríamos, en nuestras diferentes perspectivas, de tiempo y lugar. Entiendo que la teoría -o, el enfoque- de la igualdad que avanza Ruiz Miguel en Cuestiones de Principios representan la mejor expresión de una filosofía jurídica escrita en el «momento feliz» de la socialdemocracia española (o europea). Sería interesante, por ello, contrastar las conclusiones presentadas por nuestro autor, en su libro, con las perspectivas $-\mathrm{y}$, sobre todo, las continuidades, correcciones y precisiones- que hoy él podría ofrecernos al respecto.

\section{Bibliografía}

Dworkin, R. (1996). Freedom's Law. Cambridge: Harvard University Press.

Dworkin, R. (2000). Sovereign Virtue. The Theory and Practice of Equality. Cambridge: Harvard University Press.

Dworkin, R. (2006). Is democracy possible here? Principles for a new political debate. Princeton: Princeton University Press.

Ginsburg, T. \& Huq, A. (2018). How to Save a Constitutional Democracy. Chicago: The University of Chicago Press.

Graber, M., Levinson, S. \& Tushnet, M. (eds.) (2018). Constitutional democracy in crisis? Oxford: Oxford University Press.

Kymlicka, W. (2001). Review of Ronald Dworkin's Sovereign Virtue. En Isuma: Canadian Journal of Policy Research, 2/1.

Levitsky, S. \& Ziblatt, D. (2018). How Democracies Die. New York: Crown. 
Nussbaum, M. (2002). Making Philosophy Matter to Politics. The New York Times, op. ed., 2 de diciembre.

O'Neill, M. \& Williamson, T. (eds.) (2012). Property-Owning Democracy. Rawls and Beyond. Oxford: Blackwell Publ.

Rawls, J. (1971). A Theory of Justice. Cambridge: Harvard University Press.

Rawls, J. (2001). Justice as Fairness. A Restatement. Cambridge: Harvard University Prees.

Ruiz Miguel, A. (2020). Cuestiones de principios: entre política y Derecho. Madrid: Centro de Estudios Políticos y Constitucionales.

Van Parijs, P. \& Rawls, J. (2003). Three letters on The Law of Peoples and the European Union in Autour de Rawls. Special issue of Revue de philosophie économique, 7, pp. 7-20. 